\title{
MAGEC2 Correlates With Unfavorable Prognosis And Promotes Tumor Development In HCC Via Epithelial-Mesenchymal Transition
}

This article was published in the following Dove Press journal: OncoTargets and Therapy

\author{
Xuefeng $\mathrm{Gu}^{1,2, *}$ \\ Yuan $\mathrm{Mao}^{3, *}$ \\ Chuanbing $\mathrm{Shi}^{4}$ \\ Wei $\mathrm{Ye}^{2}$ \\ Ning $\mathrm{Hou}^{5}$ \\ $\mathrm{Li} \mathrm{Xu}^{5}$ \\ Yan Chen ${ }^{5}$ \\ Wei Zhao ${ }^{1,2}$
}

'Medical School, Southeast University, Nanjing, People's Republic of China; ${ }^{2}$ Department of Liver Disease, The Second Hospital of Nanjing, Medical School, Southeast University, Nanjing, People's Republic of China; ${ }^{3}$ Department of Hematology and Oncology, Geriatric Hospital of Nanjing Medical University, Jiangsu Province Geriatric Hospital, Nanjing, People's Republic of China; ${ }^{4}$ Department of Pathology, Pukou District Central Hospital, Pukou Branch of Jiangsu Province Hospital, The First Affiliated Hospital of Nanjing Medical University, Nanjing, People's Republic of China; ${ }^{5}$ Department of Pathology, Jiangsu Cancer Hospital, Affiliated Cancer Hospital of Nanjing Medical University, Nanjing, People's Republic of China

*These authors contributed equally to this work
Correspondence: Wei Zhao Medical School, Southeast University, 87 Dingjiaqiao Street, 210000 Nanjing, Jiangsu, People's Republic of China

Tel +86 $258362-6000$

Fax +86 $258362-6060$

Email weizhaoseu@I63.com
Purpose: Although MAGEC2 was first cloned from a human hepatocellular carcinoma (HCC) cDNA library by serum screening, the detailed attributes of MAGEC2 in HCC have rarely been elucidated.

Patients and methods: In this study, The Cancer Genome Atlas (TCGA) and the Gene Expression Omnibus (GEO) databases were consulted to analyse the expression of MAGEC2 mRNA in liver cancer. Immunohistochemistry (IHC) analysis was performed to detect MAGEC2 expression in HCC, and the relationship between MAGEC2 expression and the clinicopathological characteristics of HCC patients was evaluated. Then, we employed the short hairpin (sh)RNAmediated knockdown of MAGEC2 in HCC cell lines to explore the function of MAGEC2 in HCC development. Finally, the expression of epithelial-mesenchymal transition (EMT) markers in HCC xenografts and clinical samples was investigated.

Results: The results showed a remarkably higher level of MAGEC2 expression in HCC tissues than in noncancerous tissues, and MAGEC2 expression could be used as an independent prognostic factor for overall survival in HCC. Moreover, sh-MAGEC2 inhibited a series of HCC malignant behaviours both in vitro and in vivo. Finally, decreased MAGEC2 expression and low levels of EMT markers were detected in sh-MAGEC2 xenografts, while increased MAGEC2 expression and high levels of EMT markers were observed in invasive and metastatic HCC samples.

Conclusion: Taken together, our data imply that MAGEC2 is a novel prognostic marker for HCC and that MAGEC2 significantly promotes HCC tumourigenesis by inducing EMT. Targeting MAGEC2 may provide a promising therapeutic strategy for HCC treatment.

Keywords: MAGEC2, hepatocellular carcinoma, prognosis, tumourigenesis, EMT

\section{Introduction}

Hepatocellular carcinoma (HCC) has become the most common leading cause of cancer-related mortality worldwide. ${ }^{1}$ In China, the township of Qidong in Jiangsu Province is one of the highest endemic areas for HCC deaths globally. ${ }^{2}$ The tumourigenesis of $\mathrm{HCC}$ is complicated, and several factors contribute to $\mathrm{HCC}$ development, including hepatitis $\mathrm{B}$ or $\mathrm{C}$ virus infection, chronic alcohol consumption and nonalcoholic fatty liver disease. ${ }^{3}$ Although the survival rates for HCC have improved because of the development of therapeutic strategies in recent years, the overall prognosis is still unsatisfactory. ${ }^{4}$ It is highly critical to identify informative molecular biomarkers and to understand the mechanisms of hepatocarcinogenesis for HCC. 
Melanoma-associated antigens (MAGEs) represent a special type of tumour antigen that is highly expressed in tumours but weakly expressed in normal tissues. ${ }^{5}$ Because of their unique characteristics, MAGEs are recognized as ideal targets for cancer immunotherapy, and several MAGE-based immunotherapy strategies have been developed. ${ }^{6}$ MAGEC2 (HCA587) was first cloned from a human HCC cDNA library by serum screening, and it is highly expressed in several types of tumours. ${ }^{7}$ A high incidence of MAGEC2 expression has been reported in larynx squamous cell carcinoma and is remarkably correlated with an advanced clinical stage. ${ }^{8} \mathrm{~A}$ recent pilot study also reported that MAGEC2 could be a potential biomarker for triple-negative breast cancer. ${ }^{9}$ However, the detailed characteristics and mechanisms of MAGEC2 in HCC are inadequately understood.

In the present study, we first examined MAGEC2 expression in HCC tissues and evaluated its association with the clinicopathological attributes of HCC patients. Then, we constructed MAGEC2-knockdown (sh-MAGEC2) models in HCC cell lines to detect the characteristics of MAGEC2 in HCC development, both in vitro and in vivo. Finally, we explored the role of MAGEC2 in the promotion of HCC invasion and metastasis by epithelial-mesenchymal transition (EMT) detection.

\section{Materials And Methods \\ Data Acquisition}

Liver cancer mRNA expression data were downloaded from The Cancer Genome Atlas (TCGA) database (https://portal.gdc.cancer.gov/). Gene microarray data (GSE39791, GSE14520, GSE36376, GSE89377, and GSE76427) were downloaded from the Gene Expression Omnibus (GEO) database (https://www.ncbi.nlm.nih.gov/ geo/). We used $\log 2$ transformation to transform the expression level of MAGEC2 mRNA for further analysis.

\section{Tumour Tissue Specimens}

A tissue microarray (TMA) containing ninety-five HCC samples and eighty-five noncancerous samples was purchased from Outdo Biotech Co., Ltd. (Shanghai, China) to conduct IHC analysis. The original clinical data were also provided along with the TMA. Moreover, a total of 39 formaldehyde-fixed and paraffin-embedded HCC samples were collected from the Department of Pathology, Jiangsu Cancer Hospital and divided into three groups for EMT detection: the primary HCC group (13 patients), the invasive group (13 patients) and the metastatic (vascular tumour thrombus) group (13 patients). All patients provided informed consent, and the study was approved by the ethics committee of each hospital.

\section{Cells And Reagents}

One HCC cell line (MHCC97H) was generously provided by Yuanduan Biological Co., Ltd. (Jiangsu, China). Two other HCC cell lines (SK-H-1 and HepG2) and the human liver cell line LO-2 were obtained from the cell bank of the Chinese Academy of Science (Shanghai, China). For MAGEC2 knockdown, the target sequence of the short hairpin RNA (shRNA) was as follows: 5'- CCT CTT CCA CTT TGT ACT T -3' (Gene ID: NM_016249). The shRNA targeting MAGEC2 (sh-MAGEC2) was cloned into pLKD-CMVG\&PR-U6-shRNA (Obio Technology, Co., Ltd., Shanghai, China). An unrelated random sequence was used as an shMAGEC2 negative control (sh-MAGEC2-NC). After viral transduction, MAGEC2 expression was evaluated by quantitative real-time polymerase chain reaction (qPCR) and Western blotting (WB) analyses. The primary antibodies, including MAGEC2, E-cadherin, N-cadherin, fibronectin, Slug, cleaved caspase-3, Cyclin D1, Cyclin E and Ki67, were all purchased from Abcam Co., Ltd. (Abcam, Cambridge, MA, USA) and Santa Cruz Biotechnology (Santa Cruz, CA, USA).

\section{qPCR And WB Analyses}

For qPCR, total RNA was extracted using TRIzol reagent (Vazyme Biotech Co., Ltd., Jiangsu, China) according to the manufacturer's guidelines. The primers for MAGEC2 were as follows: forward primer 5'- GAA CAG TGT TGT TCA GCT TGG -3'; reverse primer 5'- GGA CAT TAC CTG GAG TAT CGG -3'. Glyceraldehyde-3-phosphate dehydrogenase (GAPDH) was used as a normalization control. For WB analysis, the detailed protocols were described in our previous research. ${ }^{10}$

\section{Immunohistochemistry (IHC) Analysis}

For IHC analysis, TMA sections were incubated with a primary monoclonal rabbit anti-MAGEC2 antibody (1:100, Abcam, Cambridge, MA, USA). The detailed protocol for IHC analysis and the evaluation of MAGEC2 expression were defined in our previous report. ${ }^{10}$ In short, the immunostaining intensity of MAGEC2 was categorized as follows: 0 (negative), 1 (weakly positive), 2 (moderately positive), and 3 (strongly positive). The immunostaining percentage of MAGEC2 was ranked as follows: $1(0-10 \%), 2(11-50 \%)$, 
$3(51-80 \%)$, and $4(81-100 \%)$. The sum of the intensity and percentage scores led to the final immunohistochemistry score (IHS). IHS $<4$ indicated low or no expression, while IHS $\geq 4$ indicated high expression.

\section{Phenotypic Experiments Of MAGEC2 In Vitro}

Cell Counting Kit-8 (CCK-8), Transwell and wound healing assays were performed in the sh-MAGEC2 HCC cell line to detect the malignant behaviours of MAGEC2, including its effects on cell proliferation, invasion and migration, as described before. ${ }^{11}$ For the apoptosis assay, HCC cells were cultured, collected and mixed with Annexin V-APC and 7-AAD (KeyGen, Co., Ltd., Jiangsu, China) for flow cytometry (FCM) detection. For the cell cycle assay, HCC cells were collected, serum starved, and cocultured with RNase A and propidium iodide (PI) (Sigma-Aldrich Chemical Company, St. Louis, MO, USA) for FCM examination. The detailed protocols of cell apoptosis and cell cycle analyses were described previously. ${ }^{12}$ Moreover, the expression of cleaved caspase-3, Cyclin D1 and Cyclin E was detected by WB analyses as described above.

\section{Tumour Growth Assay In Vivo}

Six-week-old male BALB/c nude mice were purchased from CAVENS Co., Ltd. (Changzhou, China). Equal numbers of cells $\left(5 \times 10^{6}\right)$ transfected with sh-MAGEC2 or sh-MAGEC2$\mathrm{NC}$ and untransfected MHCC97H cells were injected subcutaneously into each mouse ( $\mathrm{n}=6$ mice per group). Tumour size was recorded, and tumour volume was calculated as described previously. ${ }^{13}$ At the end of the inoculation period (day 33), all mice were sacrificed, and xenograft tumours were collected for further analyses. The protocols of in vivo experiments were approved by the Institutional Animal Care and Use Committee of Southeast University (NO. 20180706007) and in accordance with the ethical standards of the Guide for the Care and Use of Laboratory Animals of the National Institutes of Health for Animal Welfare Act. In addition, Ki67 expression in xenografts from nude mice was also examined by IHC analysis as described above.

\section{Detection Of EMT Markers By IHC And WB Analyses}

The expression of EMT markers, including E-cadherin, N-cadherin, fibronectin and Slug, was detected by IHC and WB analyses as described previously. ${ }^{11}$

\section{Statistical Analysis}

The data are expressed as the mean \pm standard deviation. Differences between 2 groups were statistically analysed using Student's $t$-test or the Mann-Whitney $U$-test. Multiple groups were compared using one-way analysis of variance (ANOVA) with least significant difference (LSD)'s post hoc correction or the Kruskal-Wallis nonparametric test followed by the Mann-Whitney $U$-test. $\mathrm{P}<$ 0.05 was considered significant. All statistical analyses were conducted using SPSS 19.0 (SPSS, Inc., Chicago, IL, USA) and STATA 12.0 (Stata Corporation, College Station, TX, USA).

\section{Results}

\section{Confirmation Of MAGEC2 Expression By} Bioinformatics Analyses

After performing bioinformatics analyses, we found that MAGEC2 mRNA expression was significantly higher in liver cancer tissues than in normal liver tissues from the TCGA cohort $(\mathrm{P}=0.000$, Figure 1A). Moreover, MAGEC2 mRNA was also overexpressed in HCC tissues compared to noncancerous tissues in the GSE39791 ( $\mathrm{P}=0.002$, Figure 1B), GSE14520 ( $\mathrm{P}=0.000$, Figure $1 \mathrm{C})$, GSE36376 $(\mathrm{P}=0.000$, Figure 1D) and GSE89377 $(\mathrm{P}=0.000$, Figure 1E) gene microarrays from the GEO cohort.

\section{Detection Of MAGEC2 Expression By IHC Analysis In The HCC TMA}

By IHC analysis, high MAGEC2 expression was detected in 45 of $95(47.4 \%)$ HCC tissues, while no MAGEC2 expression was detected in noncancerous tissues, and the difference was statistically significant $(P=0.000)$. MAGEC2 staining was localized in the nuclei of HCC cells, and representative IHC staining is illustrated in Figure 2A. High MAGEC2 expression was significantly correlated with tumour size $(\mathrm{P}=0.017)$ and prognosis $(\mathrm{P}=$ 0.009) (Table 1).

\section{Survival Analysis}

In the GSE76427 microarray from the GEO cohort, a Kaplan-Meier curve was constructed to demonstrate that the high expression of MAGEC2 mRNA was associated with unfavourable relapse-free survival (RFS) $(\mathrm{P}=0.036$, Figure 2B). In the HCC TMA, the univariate analysis revealed that MAGEC2 expression $(\mathrm{P}=0.006)$ strongly correlated with overall survival (OS) in HCC, and the multivariate analysis further indicated that MAGEC2 

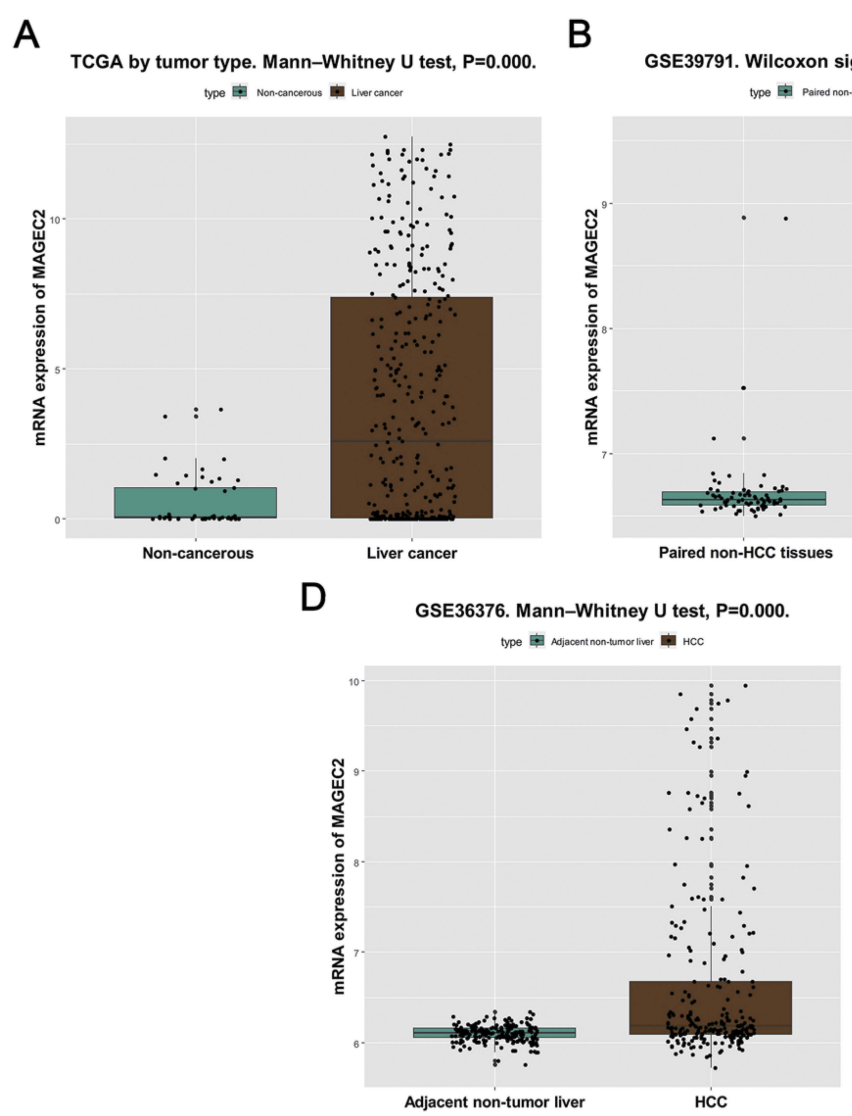

B

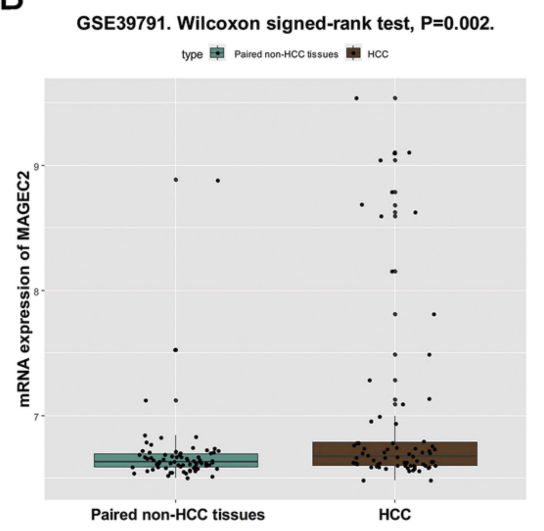

C

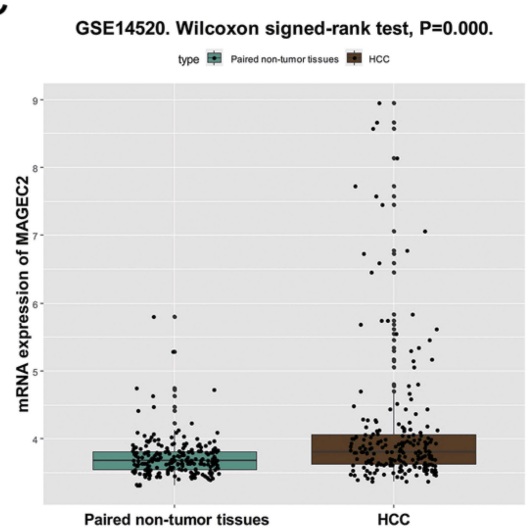

$\mathrm{E}$

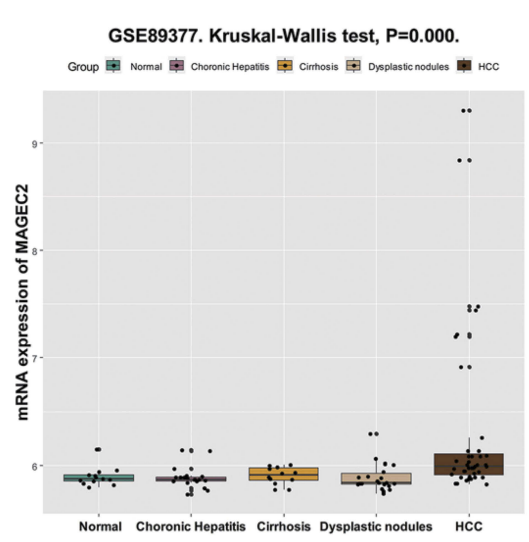

Figure I (A-E) Expression of MAGEC2 in liver cancer tissue samples by bioinformatics analyses. The MAGEC2 mRNA expression in HCC is significantly elevated compared with in normal tissues in The Cancer Genome Atlas database (A), microarrays GSE3979I (B), GSEI4520 (C), GSE36376 (D) and GSE89377 (E) (all P < 0.05 ).

expression $(\mathrm{P}=0.008)$ was an independent prognostic factor for HCC patients (Table 2). A Kaplan-Meier curve was also generated to illustrate that the overall survival was unfavourable in patients with high MAGEC2 expression compared with patients with low MAGEC2 expression $(\mathrm{P}=0.0048$, Figure $2 \mathrm{C})$.

\section{Construction Of The MAGEC2- Knockdown Model}

As shown in Figure 3A, qPCR revealed that MAGEC2 expression was significantly detected in the MHCC97H cell line but not in the LO2, HepG2 or SK-H-1 cell line. Then, a MAGEC2-knockdown model in the MHCC97H cell line was prepared by shRNA with lentiviral transfection, and the results of $\mathrm{qPCR}$ and $\mathrm{WB}$ analyses confirmed that MAGEC2 was significantly decreased in the sh-MAGEC2 group ( $\mathrm{P}=0.000$, Figure $3 \mathrm{~B}$ ). Fluorescence microscopy further showed that both the sh-MAGEC2 and control groups exhibited strong fluorescence, which validated the successful construction of sh-MAGEC2 in MHCC97H cells after lentiviral transfection for $72 \mathrm{~h}$ (Figure 3C).

\section{MAGEC2 Affects The Malignant Behaviours Of HCC In Vitro}

A series of the malignant behaviours of MAGEC2 was investigated. CCK-8 ( $P=0.000$, Figure 3D), wound healing $(\mathrm{P}=0.002$, Figure 3E) and transwell $(\mathrm{P}=0.000$, Figure $3 \mathrm{~F}$ ) assays demonstrated that sh-MAGEC2 markedly inhibited cell growth, migration and invasions in HCC. Moreover, the Annexin V-APC/7-AAD assay illustrated that sh-MAGEC2 dramatically enhanced the apoptotic rate $(\mathrm{P}=0.000$, Figure $3 \mathrm{G})$. FCM demonstrated that the $\mathrm{G} 1$ phase was significantly prolonged in sh-MAGEC2 cells $(\mathrm{P}=0.002$, Figure $3 \mathrm{H}), \mathrm{S}$ phase was significantly shortened in sh-MAGEC2 cells $(\mathrm{P}=0.001$, Figure $3 \mathrm{H})$ while $\mathrm{G} 2$ phase was rarely changed $(\mathrm{P}=0.054$, Figure $3 \mathrm{H})$, suggesting that MAGEC2 critically promotes the G1/S phase transition. Furthermore, WB analysis showed that the expression of cleaved caspase- 3 was higher in the sh-MAGEC2 group than in the sh-MAGEC2-NC group, which implies that MAGEC2 can inhibit cleaved caspase3 expression, thereby explaining the suspension of 


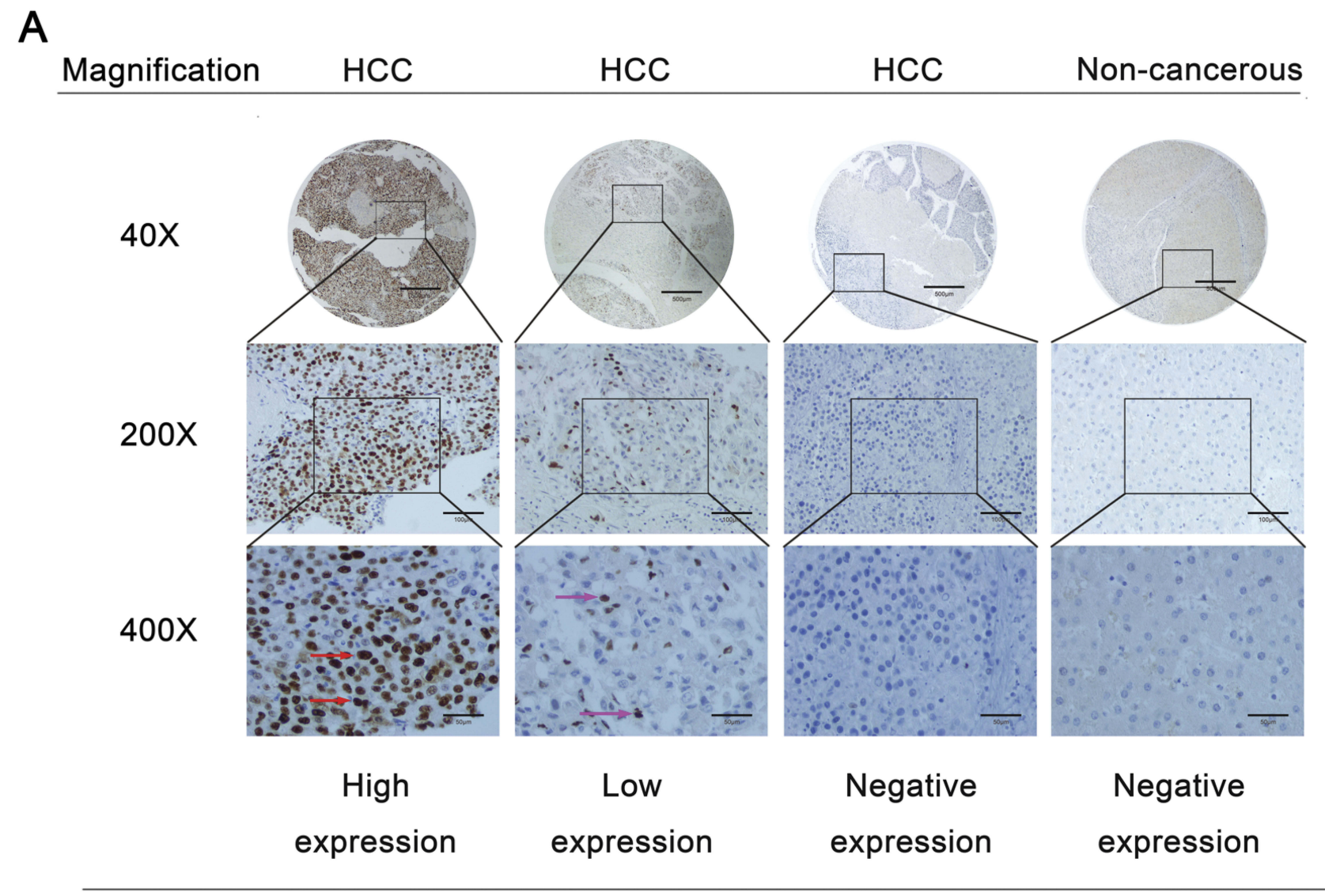

B

Kaplan-Meier curve of RFS by MAGEC2 expression in GSE76427. MAGEC2 expression + high $\mp$ low
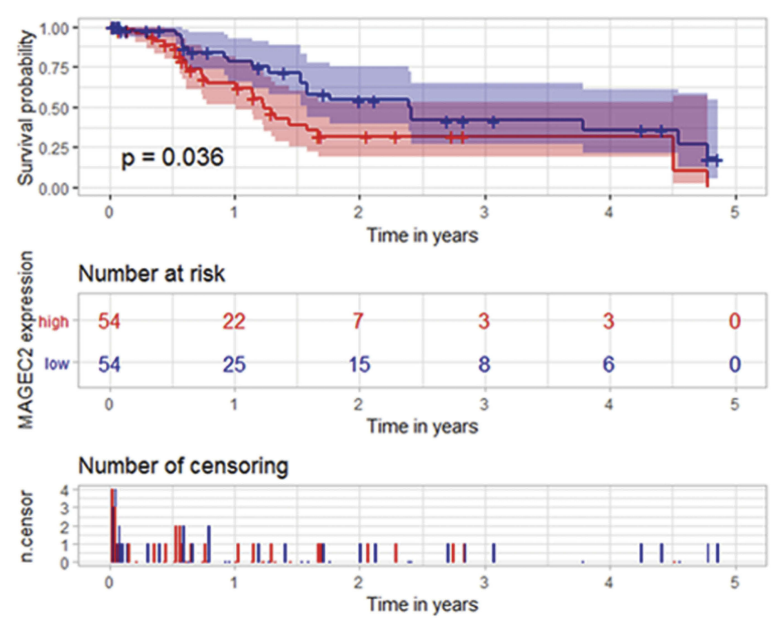

C

Kaplan-Meier curve of OS by MAGEC2 expression in HCC TMA MAGEC2 expression + high + low
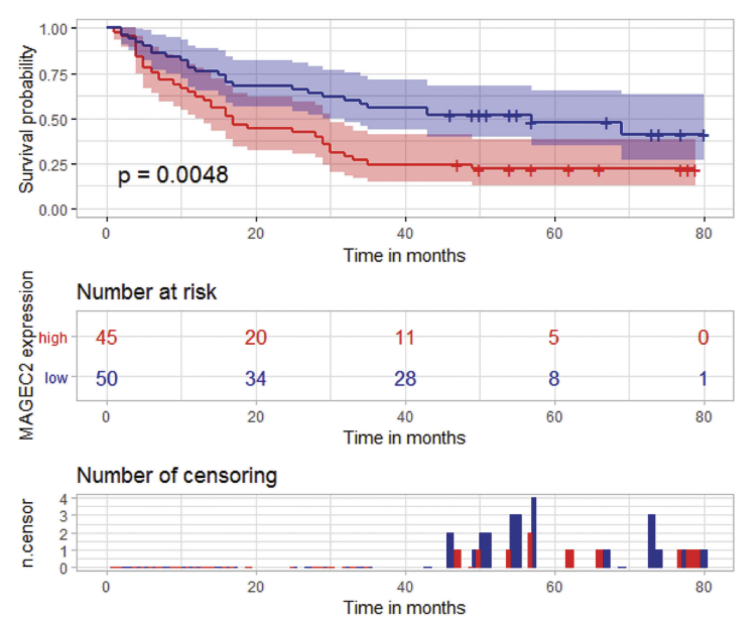

Figure 2 Survival analysis of MAGEC2 in hepatocellular carcinoma (HCC) TMA. (A) Representative images of immunohistochemical staining for MAGEC2 protein expression in HCC tissues and corresponding noncancerous tissues. Red arrows show positive staining in the nucleus of cancer cells in HCC sample with high MAGEC2 expression. Pink arrows show positive staining in the nucleus of cancer cells in HCC sample with low MAGEC2 expression. (B) Relapse-free survival rate (microarray GSE76427) in patients with high MAGEC2 expression was remarkably lower than that in patients with low MAGEC2 expression (P = 0.036). (C) Overall survival rate in patients with high MAGEC2 expression was remarkably lower than that in patients with low MAGEC2 expression in TMA (P = 0.0048).

apoptosis in HCC cells by MAGEC2 (Figure 3I). In addition, Cyclin E was downregulated while Cyclin D1 expression was unchanged in the sh-MAGEC2 group, which indicates that Cyclin E might participate in the cell cycle modulation by MAGEC2 in the G1-S transition in HCC cells (Figure 3I). 
Table I Relationship Of High MAGEC2 Expression With Clinicopathological Characteristics In HCC

\begin{tabular}{|c|c|c|c|c|c|}
\hline \multirow[t]{2}{*}{ Groups } & \multirow[t]{2}{*}{ No. } & \multicolumn{2}{|c|}{ MAGEC2 } & \multirow[t]{2}{*}{$\chi^{2}$} & \multirow[t]{2}{*}{ p Value } \\
\hline & & + & $\%$ & & \\
\hline \multicolumn{6}{|l|}{ Gender } \\
\hline Male & 85 & 42 & 49.4 & 1.352 & 0.245 \\
\hline Female & 10 & 3 & 30.0 & & \\
\hline \multicolumn{6}{|l|}{ Age (years) } \\
\hline$<60$ & 70 & 31 & 44.3 & 1.413 & 0.235 \\
\hline$\geq 60$ & 24 & 14 & 58.3 & & \\
\hline Insufficient data & 1 & 0 & & & \\
\hline \multicolumn{6}{|l|}{ Tumor size $(\mathrm{cm})$} \\
\hline$<5$ & 39 & 13 & 33.3 & 5.646 & $0.017^{*}$ \\
\hline$\geq 5$ & 55 & 32 & 58.2 & & \\
\hline Insufficient data & 1 & 0 & & & \\
\hline \multicolumn{6}{|l|}{ Pathological grade } \\
\hline I-II & 61 & 29 & 47.5 & 0.002 & 0.964 \\
\hline III & 34 & 16 & 47.1 & & \\
\hline \multicolumn{6}{|l|}{$\mathrm{T}$} \\
\hline $\mathrm{TI}$ & 12 & 3 & 25.0 & 6.746 & 0.080 \\
\hline $\mathrm{T} 2$ & 31 & 12 & 38.7 & & \\
\hline T3 & 41 & 21 & 51.2 & & \\
\hline $\mathrm{T} 4$ & 3 & 3 & 100.0 & & \\
\hline Insufficient data & 8 & 6 & & & \\
\hline \multicolumn{6}{|l|}{ Liver cirrhosis } \\
\hline Yes & 36 & 16 & 44.4 & 0.199 & 0.656 \\
\hline No & 59 & 29 & 49.2 & & \\
\hline \multicolumn{6}{|l|}{ Gross type } \\
\hline Diffuse & 2 & 1 & 50.0 & 7.684 & 0.053 \\
\hline Nodular & 38 & 13 & 34.2 & & \\
\hline Multinodular & 6 & 4 & 66.7 & & \\
\hline Massive & 25 & 17 & 68.0 & & \\
\hline Insufficient data & 24 & 10 & & & \\
\hline \multicolumn{6}{|l|}{$\mathrm{N}$} \\
\hline No & 86 & 38 & 44.2 & 1.245 & 0.264 \\
\hline $\mathrm{NI}$ & 1 & I & 100.0 & & \\
\hline Insufficient data & 8 & 6 & & & \\
\hline \multicolumn{6}{|l|}{ M } \\
\hline Mo & 87 & 39 & 44.8 & 1.214 & $0.27 I$ \\
\hline MI & 1 & I & 100.0 & & \\
\hline Insufficient data & 7 & 5 & & & \\
\hline \multicolumn{6}{|l|}{ TNM stage } \\
\hline Stage I & 12 & 3 & 25.0 & 5.807 & 0.121 \\
\hline Stage II & 31 & 12 & 38.7 & & \\
\hline Stage III & 42 & 22 & 52.4 & & \\
\hline Stage IV & 2 & 2 & 100.0 & & \\
\hline Insufficient data & 8 & 6 & & & \\
\hline
\end{tabular}

(Continued) 
Table I (Continued).

\begin{tabular}{|c|c|c|c|c|c|}
\hline \multirow[t]{2}{*}{ Groups } & \multirow[t]{2}{*}{ No. } & \multicolumn{2}{|c|}{ MAGEC2 } & \multirow[t]{2}{*}{$\chi^{2}$} & \multirow[t]{2}{*}{ p Value } \\
\hline & & + & $\%$ & & \\
\hline \multicolumn{6}{|l|}{ Survival } \\
\hline Positive & 34 & 10 & 29.4 & 6.848 & $0.009 *$ \\
\hline Negative & 61 & 35 & 57.4 & & \\
\hline
\end{tabular}

Note: $* P<0.05$

Abbreviations: $\mathrm{HCC}$, hepatocellular carcinoma; $\mathrm{T}$, tumor stage; $\mathrm{N}$, node; $\mathrm{M}$, metastasis.

Table 2 Univariate And Multivariate Analysis Of Prognostic Factors In HCC For Overall Survival

\begin{tabular}{|c|c|c|c|c|c|c|}
\hline & \multicolumn{3}{|c|}{ Univariate Analysis } & \multicolumn{3}{|c|}{ Multivariate Analysis } \\
\hline & HR & P Value & $95 \% \mathrm{Cl}$ & HR & P Value & $95 \% \mathrm{Cl}$ \\
\hline \multicolumn{7}{|l|}{ MAGEC2 expression } \\
\hline High versus Low & 2.04 & $0.006 *$ & $1.222-3.398$ & 2.59 & $0.008^{*}$ & $1.285-5.228$ \\
\hline \multicolumn{7}{|l|}{ Gender } \\
\hline Male versus Female & 1.49 & 0.390 & $0.598-3.732$ & & & \\
\hline \multicolumn{7}{|l|}{ Age (years) } \\
\hline$<60$ versus $\geq 60$ & 0.63 & 0.140 & $0.34 I-1.164$ & & & \\
\hline \multicolumn{7}{|l|}{ Tumor size $(\mathrm{cm})$} \\
\hline$<5$ versus $\geq 5$ & 0.41 & $0.002^{*}$ & $0.235-0.721$ & 0.54 & 0.200 & $0.212-1.382$ \\
\hline \multicolumn{7}{|l|}{ Pathological grade } \\
\hline I-II versus III & 0.74 & 0.254 & $0.443-1.240$ & & & \\
\hline \multicolumn{7}{|l|}{ Liver cirrhosis } \\
\hline Yes versus No & 0.96 & 0.887 & $0.573-1.619$ & & & \\
\hline \multicolumn{7}{|l|}{ Gross type } \\
\hline Diffuse versus Nodular versus Multinodular versus Massive & 0.73 & $0.046^{*}$ & $0.542-0.994$ & 0.84 & 0.400 & $0.570-1.252$ \\
\hline \multicolumn{7}{|l|}{$\mathrm{T}$} \\
\hline TI versus $\mathrm{T} 2$ versus $\mathrm{T} 3$ versus $\mathrm{T} 4$ & 0.48 & $0.000^{*}$ & $0.319-0.708$ & 0.63 & 0.385 & $0.227-1.773$ \\
\hline \multicolumn{7}{|l|}{$\mathrm{N}$} \\
\hline No versus $\mathrm{NI}$ & 0.34 & 0.289 & $0.046-2.501$ & & & \\
\hline \multicolumn{7}{|l|}{ M } \\
\hline MO versus $\mathrm{MI}$ & 0.02 & $0.002 *$ & $0.002-0.255$ & 0.10 & 0.091 & $0.006-1.448$ \\
\hline \multicolumn{7}{|l|}{ TNM stage } \\
\hline I versus II versus III versus IV & 0.45 & $0.000 *$ & $0.294-0.692$ & 0.45 & 0.212 & $0.128-1.576$ \\
\hline
\end{tabular}

Note: $* \mathrm{P}<0.05$

Abbreviations: $\mathrm{HR}$, hazard ratio; $\mathrm{Cl}$, Confidence interval.

\section{MAGEC2 Facilitates The Xenograft Growth Of HCC In Vivo}

To investigate the function of MAGEC2 in HCC development, we used three cell lines (MHCC97H untransfected, sh-MAGEC2 and sh-MAGEC2-NC) to construct HCC xenografts in nude mice. As shown in Figure 4A, the size of xenograft tumours in the sh-MAGEC2 group was remarkably smaller than those from the sh-MAGEC2-NC group or the MHCC97H untransfected group on day 33 . Tumour volume curves and tumour weight comparisons also demonstrated that tumour growth was significantly decreased in the sh-MAGEC2 group compared with the sh-MAGEC2-NC group or the MHCC97H untransfected group $(\mathrm{P}=0.002$ and $\mathrm{P}=0.003$, Figure $4 \mathrm{~B}$ and $\mathrm{C}$, 
A
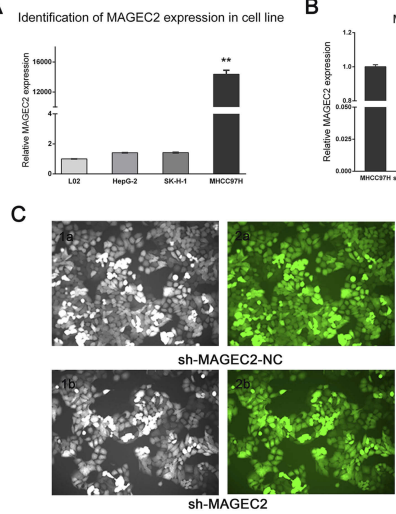

B
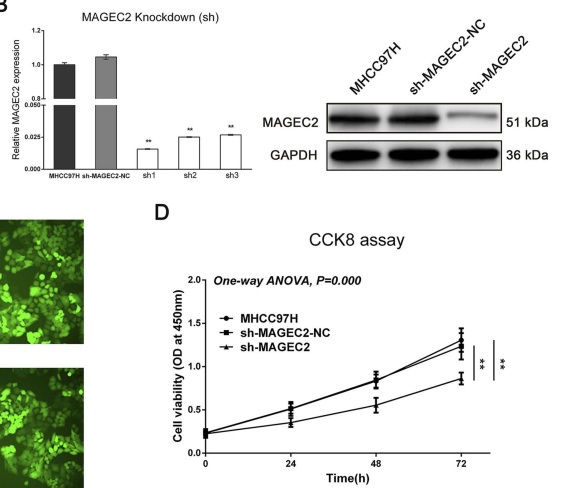

E

MHCC97H Sh-MAGEC2-NC Sh-MAGEC2
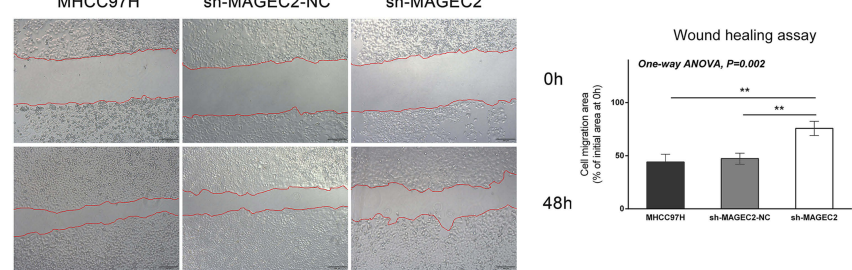

F
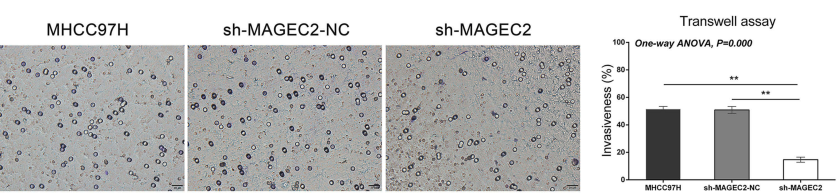

$\mathrm{G}_{\text {(a) }}$

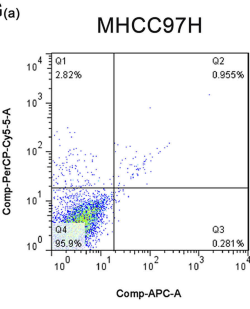

sh-MAGEC2-NC

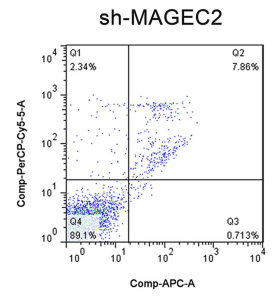

$H_{(a)}$

$\mathrm{MHCC} 97 \mathrm{H}$

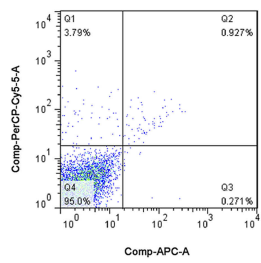

sh-MAGEC2-NC

sh-MAGEC2
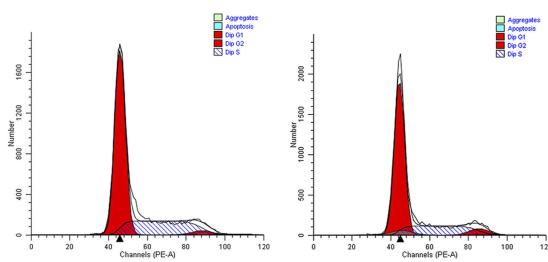

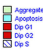

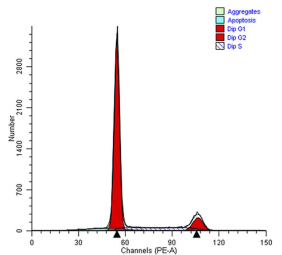

$\mathbf{G}_{(b)}$

$H_{(b)}$
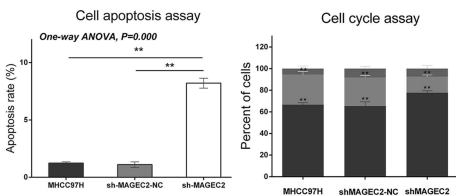

I
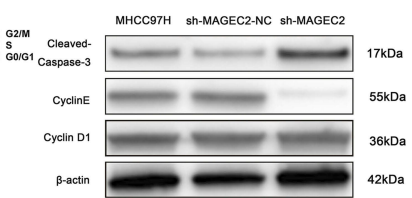

Figure 3 (A) qPCR test confirmed high MAGEC2 expression in MHCC97H cell line, but not in LO2, HepG-2 and SK-H-I cell lines. (B) qPCR test and Western blotting analysis confirmed the effectiveness of sh-MAGEC2. MAGEC2 expression was significantly inhibited in the sh-MAGEC2 group but not in the MHCC97H group or sh-MAGEC2-NC group. (C) Fluorescence microscope showed that both the sh-MAGEC2 and sh-MAGEC2-NC groups exhibited positive fluorescence, which validated the successful construction of sh-MAGEC2 or sh-MAGEC2-NC after lentiviral transfection. Fluorescence Magnification $\times 200$. (D) CCK8 assay showed that sh-MAGEC2 significantly decreased cell viability. (E) Wound healing assay showed that sh-MAGEC2 critically decreased cell migration. (F) Transwell assay showed that sh-MAGEC2 critically deceased cell invasiveness. (G(a, b)) FCM results showed that sh-MAGEC2 critically increased cell apoptosis. (H(a, b)) FCM results showed that sh-MAGEC2 significantly prolonged the GI phase and shortened the S phase. (I) Western blotting analyses showed that sh-MAGEC2 significantly elevated cleaved-caspase-3 expression and down-regulated Cyclin E expression. In comparison, there was no significant change for CyclinDI by in shMAGEC2 group. $* * \mathrm{P}<0.01$ 
A

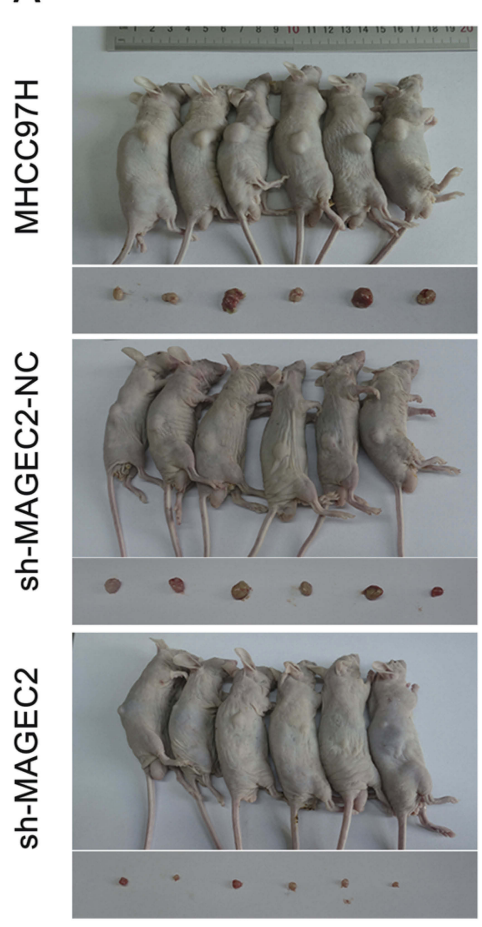

B

\section{sers}
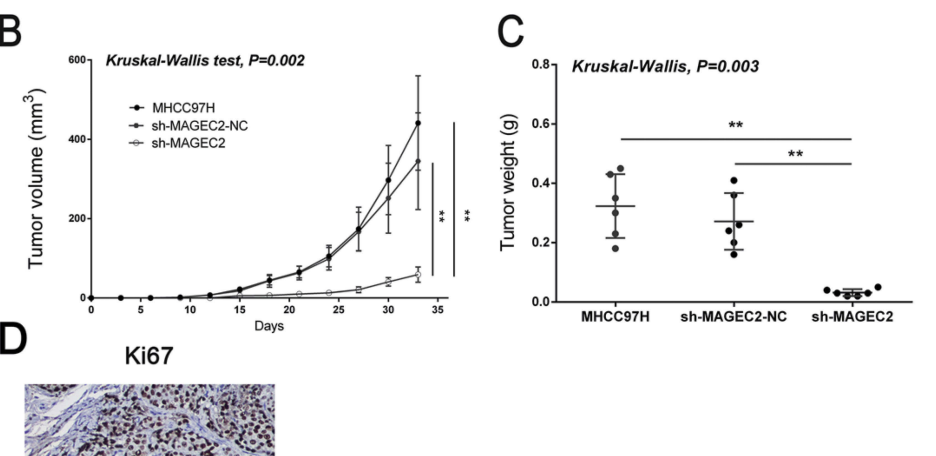

$\mathrm{MHCC} 97 \mathrm{H}$

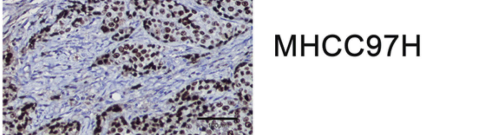

$\mathrm{E}$

sh-MAGEC2-NC

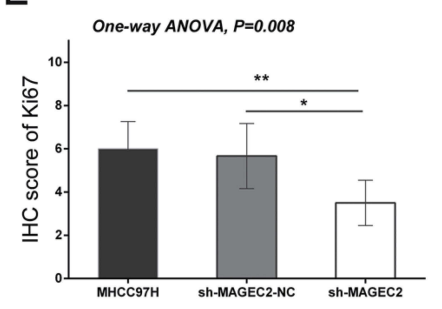

Figure 4 (A) After inoculation for 33 days, the growth of xenograft tumors in sh-MAGEC2 group was significantly inhibited compared with MHCC97H group and shMAGEC2-NC group. (B) The tumor growth curve illustrated the significantly lower rate of tumor growth in the sh-MAGEC2 group. (C) The tumor weight in sh-MAGEC2 group was significantly lower than that of in MHCC97H group and sh-MAGEC2-NC group. (D, E) IHC analysis showed that MAGEC2 knockdown significantly inhibited the expression of $\mathrm{Ki}-67$. $* * \mathrm{P}<0.01$. $* \mathrm{P}<0.05$. IHC Magnification $\times 400$.

respectively). The expression of $\mathrm{Ki} 67$ in xenografts from nude mice was then detected by IHC analysis and demonstrated a significant reduction in the sh-MAGEC2 group, which further implied the stimulating role of MAGEC2 in tumour growth (Figure 4D and E). All the above data strongly suggest that MAGEC2 dramatically enhances HCC development.

\section{MAGEC2 Promotes HCC Invasion And Metastasis Via EMT}

In the sh-MAGEC2 group, the results of IHC and WB analyses demonstrated that the expression of the epithelial marker E-cadherin $(\mathrm{P}=0.009)$ was significantly elevated, whereas the expression levels of MAGEC2 $(\mathrm{P}=0.007)$ and the mesenchymal markers $\mathrm{N}$-cadherin $(\mathrm{P}=0.000)$, fibronectin $(P=0.004)$, and Slug $(P=0.003)$ were remarkably decreased compared to the sh-MAGEC2-NC group or the $\mathrm{MHCC} 97 \mathrm{H}$ untransfected group (Figure 5A, C and $\mathrm{E}$ ). Moreover, invasive and metastatic (vascular tumour thrombus) HCC samples exhibited reduced E-cadherin expression $(\mathrm{P}=0.026)$ but increased MAGEC2 $(\mathrm{P}=0.001), \mathrm{N}$-cadherin $(\mathrm{P}=0.003)$, fibronectin $(\mathrm{P}=0.012)$, and Slug $(\mathrm{P}=0.027)$ expression compared to primary $\mathrm{HCC}$ samples (Figure $5 \mathrm{~B}$ and D). The above data imply that MAGEC2 promotes HCC invasion and metastasis via EMT.

\section{Discussion}

Although there are racial and regional differences in MAGEC2 expression, it is well acknowledged that targeting MAGEC2 has enormous potential in immunotherapy, and several MAGEC2-based immunotherapeutic strategies have been reported. For instance, Sideras et al $^{14}$ developed a panel containing four tumour antigens (MAGEC1, MAGEC2, GPC-3 and Annexin) that may be suitable for vaccination studies in HCC patients in western, low-endemic areas. However, in eastern Asia, targeting MAGEC2 may provide a promising strategy for $\mathrm{HCC}$ treatment because of the predominant expression of MAGEC2. ${ }^{14-18}$ However, the detailed characteristics of MAGEC2 in HCC tumourigenesis, as well as the underlying mechanisms, deserve further exploration.

In the present study, after bioinformatics acquisition and IHC analysis, we found elevated MAGEC2 expression in HCC at both the mRNA and protein levels. Additionally, MAGEC2 expression was closely correlated with certain malignant behaviours in HCC patients, 

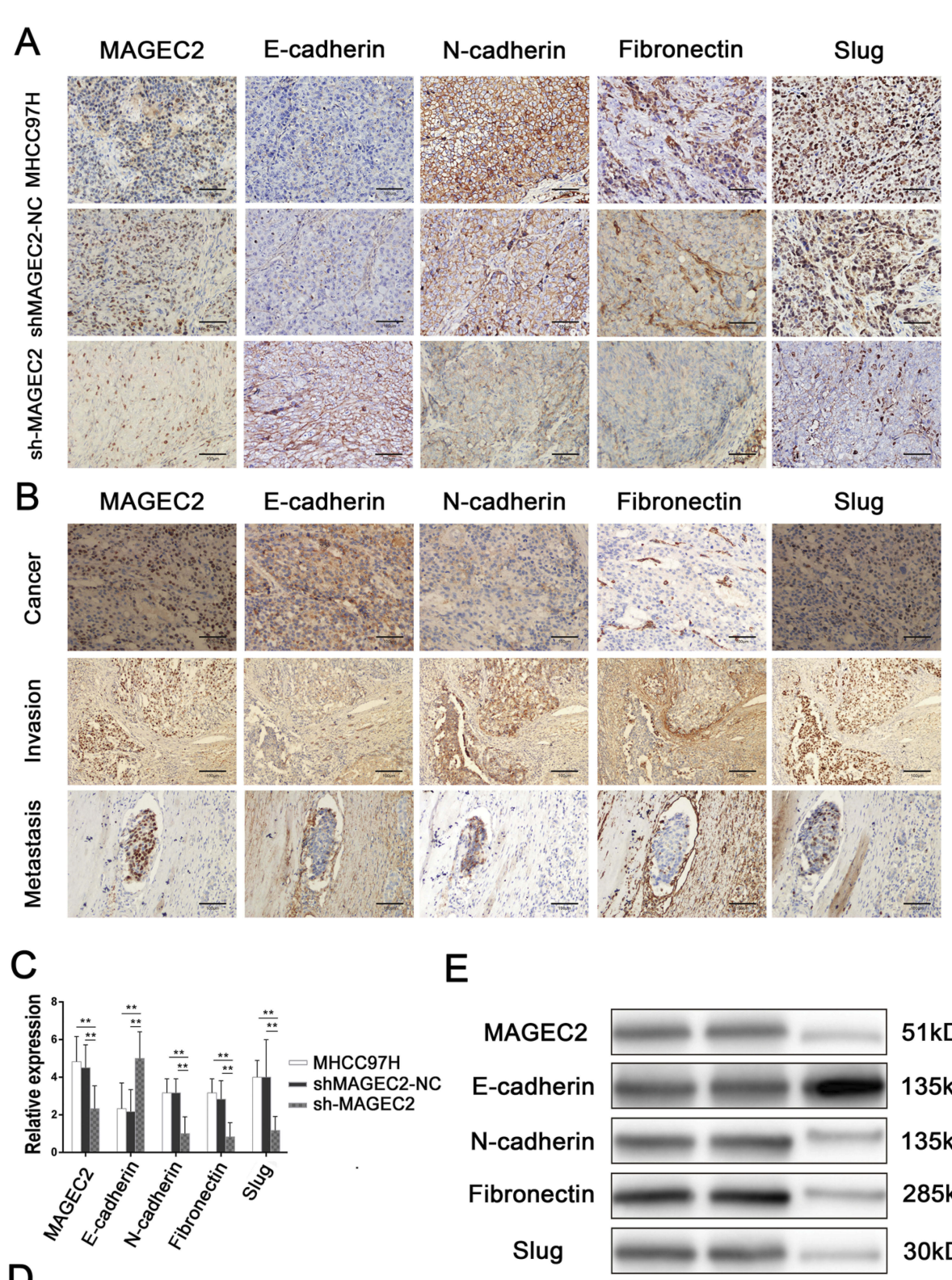

$\mathrm{E}$
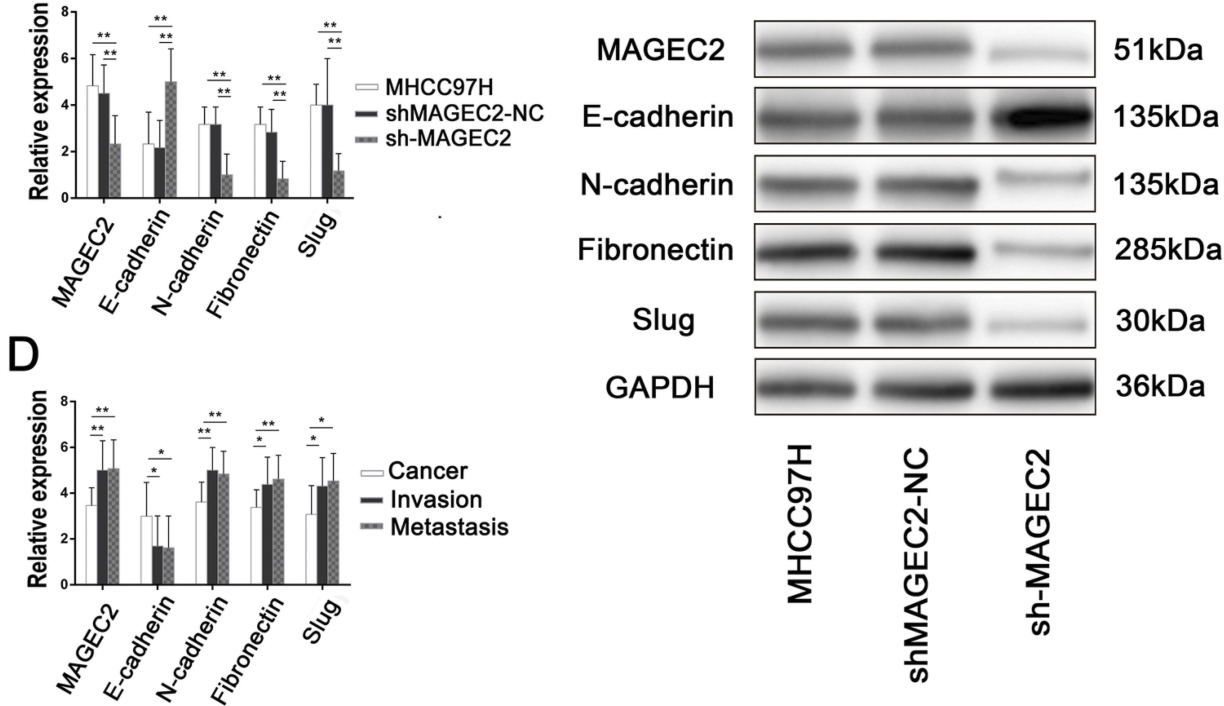

Figure 5 (A, C) Downregulation of MAGEC2 reduces EMT in HCC cells. IHC analysis of xenograft tumors confirmed the reduced expression of MAGEC2 in shMAGEC2 group. The expression of mesenchymal markers $\mathrm{N}$-cadherin, fibronectin and Slug was decreased while the expression of the epithelial marker E-cadherin was elevated in sh-MAGEC2 group compared to MHCC97H group and sh-MAGEC2-NC group. $* * P<0.01$. (B, D) IHC analyses in clinical invasive and metastatic (vascular tumor thrombus) HCC samples showed increased MAGEC2 expression, decreased E-cadherin expression, and increased N-cadherin, fibronectin, and Slug expression compared to primary HCC tissue samples.**P<0.01.*P<0.05. IHC Magnification $\times 400$. (E) Western blotting analysis further demonstrated that after MAGEC2 was downregulated, the expression of E-cadherin was significantly increased, while the expression of MAGE-C2, N-cadherin, fibronectin, and Slug was significantly decreased. 
including tumour size, OS and RFS. The survival analysis further demonstrated that MAGEC2 expression is an independent prognostic factor for $\mathrm{HCC}$ patients. Collective studies support the notion that elevated MAGEC2 expression can be observed in different cancers ${ }^{19,20}$ and that MAGEC2 expression is correlated with several important clinicopathological attributes, including tumour size, ${ }^{21}$ TNM stage, ${ }^{8}$ and recurrence. ${ }^{22}$ Moreover, Zhao et $\mathrm{al}^{9}$ and Figueiredo et $\mathrm{al}^{8}$ described the prognostic role of MAGEC2 in breast cancer and larynx cancer, respectively. Our data are in accordance with the findings of these previous studies.

Then, we examined MAGEC2 expression in different HCC cell lines and successfully constructed sh-MAGEC2 models to further explore the function of MAGEC2 in HCC development. A series of in vitro phenotypic experiments demonstrated that MAGEC2 could promote HCC development in several ways, including augmenting proliferation, increasing migration, reinforcing invasion, inhibiting apoptosis and facilitating G1/S phase transition, by inhibiting cleaved caspase-3 expression and elevating Cyclin E expression.

Subsequently, we employed nude mice to further confirm that MAGEC2 can promote HCC development in vivo. Ki67 expression in nude mice was examined, and the results demonstrated that $\mathrm{Ki} 67$ expression was significantly reduced after MAGEC2 knockdown, which further implied the facilitating role of MAGEC2 in tumour growth. Similarly, Bhatia et $\mathrm{al}^{23}$ showed that MAGEC2 promoted tumour growth and tumourigenicity in melanoma; Hao et $\mathrm{al}^{24}$ revealed that MAGEC2 overexpression increased the G1-S transition and cell proliferation in multiple kinds of cancer, including colorectal cancer, cervical cancer, breast cancer and melanoma; Jiang et $\mathrm{al}^{25}$ stated that MAGEC2 promoted proliferation, migration, and invasion and suppressed apoptosis in non-small cell lung carcinoma (NSCLC) cell lines, and MAGEC2 could also facilitate tumour growth and metastasis in NSCLC xenografts.

EMT is a multistep biological process in which epithelial cells alter in plasticity and transition to a mesenchymal phenotype; ${ }^{26}$ hence, EMT is significantly associated with the motility, invasion, and metastasis of tumour cells. ${ }^{27}$ Emerging evidence suggests that cancer/testis antigens (CTAs) can promote EMT and the genesis of cancer stem-like cells, escalating tumourigenesis, invasion, and metastasis. ${ }^{28}$ As an important member of the CTA family, it is rational to assume that MAGEC2 may exert an oncogenic role in HCC development via EMT. Our EMT detection data showed that sh-MAGEC2 increased the expression of the epithelial marker E-cadherin and decreased the expression levels of the mesenchymal markers N-cadherin, fibronectin, and Slug. Further IHC analyses in clinically invasive and metastatic HCC samples also suggested that MAGEC2 promoted HCC invasion and metastasis via EMT. Similarly, Jiang et $\mathrm{al}^{25}$ recently reported that MAGEC2 upregulated the expression of EMT-related proteins and enhanced growth and metastasis in NSCLC; Chen et $\mathrm{al}^{29}$ stated that MAGEC2 facilitated colony formation and migration by inducing the EMT of tumour cells in oesophageal squamous cell carcinoma; Yang et $\mathrm{al}^{30}$ described that MAGEC2 acted as an EMT inducer and was dramatically associated with breast cancer metastasis. Our present results are consistent with previous studies on the EMT-promoting role of MAGEC2 in human cancers.

There are several limitations to this study. First, the quality of the TMA is mediocre because the numbers of positive samples of lymph node metastases and distant metastases are small, and some important clinical information for $\mathrm{HCC}$ is missing (i.e., $\mathrm{HBV} / \mathrm{HCV}$ infection, alcohol consumption, and vascular invasion). We believe that this is the main reason that we did not identify substantial relationships between MAGEC2 expression and lymph node and distant metastases. Second, we did not construct a lung metastasis model of HCC. However, a number of preliminary experiments concerning lung metastases are ongoing, including selection of the mouse type, confirmation of the cell loading position, and the identification of cell number and status. Third, we failed to examine the second HCC cell line with relatively low MAGEC2 expression for the purpose of constructing a MAGEC2 overexpression model, which could have strengthened the power of the present results. The main reason for this was that we did not identify an HCC cell line with low MAGEC2 expression. Fourth, the exact mechanism of MAGEC2 in HCC development remains obscure. In the present study, we found that MAGEC2 facilitated HCC development by increasing Cyclin E expression and inhibiting cleaved caspase-3 expression. We simultaneously observed that MAGEC2 could promote HCC tumourigenesis by inducing EMT. Hence, we suspect that there are deep relationships between apoptosis and EMT caused by MAGEC2 in HCC development. Two arrays, including the Cancer Phospho Explorer Antibody Array and the AKT Signalling Pathway Antibody Array, have been purchased 
from Wayen Bio Co., Ltd. (Shanghai, China), and related signalling pathway experiments are ongoing.

In summary, we demonstrated for the first time that elevated MAGEC2 expression was remarkably correlated with an unfavourable prognosis in HCC, and MAGEC2 might be identified as a novel independent prognostic factor for HCC. Moreover, MAGEC2 could enhance HCC development both in vitro and in vivo by inducing EMT. Our findings highlight the potential of MAGEC2 as a promising therapeutic target for HCC treatment.

\section{Conclusion}

In conclusion, this study is the first to report that high MAGEC2 expression is correlated with unfavourable survival in HCC patients and that MAGEC2 might be used as a prognostic factor for HCC. Moreover, MAGEC2 could promote HCC development by inducing EMT. These findings indicate that targeting MAGEC2 provides a potential strategy for HCC treatment.

\section{Disclosure}

The authors report no conflicts of interest in this work.

\section{References}

1. Miller KD, Siegel RL, Lin CC, et al. Cancer treatment and survivorship statistics, 2016. CA Cancer J Clin. 2016;66(4):271-289. doi:10.3322/caac. 21349

2. $\mathrm{Fu} \mathrm{M}, \mathrm{Gu} \mathrm{X}, \mathrm{Ni} \mathrm{H}$, et al. High expression of inositol polyphosphate phosphatase-like 1 associates with unfavorable survival in hepatocellular carcinoma. Int J Clin Exp Pathol. 2013;6(11):25152522.

3. Ding J, Wang H. Multiple interactive factors in hepatocarcinogenesis. Cancer Lett. 2014;346(1):17-23. doi:10.1016/j.canlet.2013.12.024

4. Zheng H, Yang Y, Han J, et al. TMED3 promotes hepatocellular carcinoma progression via IL-11/STAT3 signaling. Sci Rep. 2016;6:37070. doi:10.1038/srep37070

5. Weon JL, Potts PR. The MAGE protein family and cancer. Curr Opin Cell Biol. 2015;37:1-8. doi:10.1016/j.ceb.2015.08.002

6. Cameron BJ, Gerry AB, Dukes J, et al. Identification of a Titinderived HLA-A1-presented peptide as a cross-reactive target for engineered MAGE A3-directed T cells. Sci Transl Med. 2013;5 (197):197ra103. doi:10.1126/scitranslmed.3006034

7. Wang Y, Han KJ, Pang XW, et al. Large scale identification of human hepatocellular carcinoma-associated antigens by autoantibodies. $J$ Immunol. 2002;169(2):1102-1109. doi:10.4049/jimmunol.169.7.4046

8. Figueiredo DL, Mamede RC, Spagnoli GC, et al. High expression of cancer testis antigens MAGE-A, MAGE-C1/CT7, MAGE-C2/CT10, NY-ESO-1, and gage in advanced squamous cell carcinoma of the larynx. Head Neck. 2011;33(5):702-707. doi:10.1002/hed.21522

9. Zhao Q, Xu WT, Shalieer T. Pilot study on MAGE-C2 as a potential biomarker for triple-negative breast cancer. Dis Markers. 2016;2016:2325987. doi:10.1155/2016/2325987

10. Gu X, Fu M, Ding Y, et al. TIMP-3 expression associates with malignant behaviors and predicts favorable survival in HCC. PLoS One. 2014;9(8):e106161. doi:10.1371/journal.pone.0106161
11. Zhang L, Mao Y, Mao Q, et al. FLOT1 promotes tumor development, induces epithelial-mesenchymal transition, and modulates the cell cycle by regulating the Erk/Akt signaling pathway in lung adenocarcinoma. Thorac Cancer. 2019;10(4):909-917. doi:10.1111/17597714.13027

12. Shi X, Ran L, Liu Y, et al. Knockdown of hnRNP A2/B1 inhibits cell proliferation, invasion and cell cycle triggering apoptosis in cervical cancer via PI3K/AKT signaling pathway. Oncol Rep. 2018;39 (3):939-950.

13. Mao Y, Zhang DW, Wen J, et al. A novel LMP1 antibody synergizes with mitomycin $\mathrm{C}$ to inhibit nasopharyngeal carcinoma growth in vivo through inducing apoptosis and downregulating vascular endothelial growth factor. Int J Mol Sci. 2012;13(2):2208-2218. doi:10.3390/ijms 13022208

14. Sideras K, Bots SJ, Biermann K, et al. Tumour antigen expression in hepatocellular carcinoma in a low-endemic western area. $\mathrm{Br} J$ Cancer. 2015;112(12):1911-1920. doi:10.1038/bjc.2015.92

15. Ma W, Vigneron N, Chapiro J, et al. A MAGE-C2 antigenic peptide processed by the immunoproteasome is recognized by cytolytic $\mathrm{T}$ cells isolated from a melanoma patient after successful immunotherapy. Int J Cancer. 2011;129(10):2427-2434. doi:10.1002/ijc.25911

16. Zhang L, Chen J, Song X, et al. Cancer/testis antigen HCA587derived long peptide vaccine generates potent immunologic responses and antitumor effects in mouse model. Oncol Res. 2013;21(4):193-200. doi:10.3727/096504014X13887748696789

17. Wen W, Zhang L, Peng J, et al. Identification of promiscuous HLADR-restricted CD4(+) T-cell epitopes on the cancer-testis antigen HCA587. Cancer Sci. 2011;102(8):1455-1461. doi:10.1111/j.13497006.2011.01930.x

18. Riener MO, Wild PJ, Soll C, et al. Frequent expression of the novel cancer testis antigen MAGE-C2/CT-10 in hepatocellular carcinoma. Int J Cancer. 2009;124(2):352-357. doi:10.1002/ijc.24229

19. Laban S, Giebel G, Klumper N, et al. MAGE expression in head and neck squamous cell carcinoma primary tumors, lymph node metastases and respective recurrences-implications for immunotherapy. Oncotarget. 2017;8(9):14719-14735. doi:10.18632/oncotarget.14830

20. Hou S, Sang M, Zhao L, Hou R, Shan B. The expression of MAGE$\mathrm{C} 1$ and MAGE-C2 in breast cancer and their clinical significance. $\mathrm{Am}$ J Surg. 2016;211(1):142-151. doi:10.1016/j.amjsurg.2015.05.028

21. Ghadban T, Perez DR, Vashist YK, et al. Expression of cancer testis antigens CT10 (MAGE-C2) and GAGE in gastrointestinal stromal tumors. Eur J Surg Oncol. 2014;40(10):1307-1312. doi:10.1016/j. ejso.2014.03.011

22. von Boehmer L, Keller L, Mortezavi A, et al. MAGE-C2/CT10 protein expression is an independent predictor of recurrence in prostate cancer. PLoS One. 2011;6(7):e21366. doi:10.1371/journal. pone. 0021366

23. Bhatia N, Xiao TZ, Rosenthal KA, et al. MAGE-C2 promotes growth and tumorigenicity of melanoma cells, phosphorylation of KAP1, and DNA damage repair. J Invest Dermatol. 2013;133(3):759-767. doi:10.1038/jid.2012.355

24. Hao J, Song X, Wang J, et al. Cancer-testis antigen MAGE-C2 binds Rbx1 and inhibits ubiquitin ligase-mediated turnover of cyclin E. Oncotarget. 2015;6(39):42028-42039. doi:10.18632/oncotarget.5973

25. Jiang S, Liu X, Li D, et al. Study on attenuating angiogenesis and epithelial-mesenchymal transition (EMT) of non-small cell lung carcinoma (NSCLC) by regulating MAGEC2. Technol Cancer Res Treat. 2018;17:1533033818797587. doi:10.1177/1533033818797587

26. Li L, Li W. Epithelial-mesenchymal transition in human cancer: comprehensive reprogramming of metabolism, epigenetics, and differentiation. Pharmacol Ther. 2015;150:33-46. doi:10.1016/j. pharmthera.2015.01.004

27. Antognelli C, Cecchetti R, Riuzzi F, Peirce MJ, Talesa VN. Glyoxalase 1 sustains the metastatic phenotype of prostate cancer cells via EMT control. J Cell Mol Med. 2018;22(5):2865-2883. doi:10.1111/jcmm.2018.22.issue-5 
28. Yang $\mathrm{P}$, Huo Z, Liao H, Zhou Q. Cancer/testis antigens trigger epithelial-mesenchymal transition and genesis of cancer stem-like cells. Curr Pharm Des. 2015;21(10):1292-1300. doi:10.2174/ 1381612821666141211154707

29. Chen X, Wang L, Yue D, et al. Correlation between the high expression levels of cancer-germline genes with clinical characteristics in esophageal squamous cell carcinoma. Histol Histopathol. 2017;32(8):793-803.
30. Yang F, Zhou X, Miao X, et al. MAGEC2, an epithelial-mesenchymal transition inducer, is associated with breast cancer metastasis. Breast Cancer Res Treat. 2014;145(1):23-32. doi:10.1007/s10549014-2915-9

\section{Publish your work in this journal}

OncoTargets and Therapy is an international, peer-reviewed, open access journal focusing on the pathological basis of all cancers, potential targets for therapy and treatment protocols employed to improve the management of cancer patients. The journal also focuses on the impact of management programs and new therapeutic

Submit your manuscript here: https://www.dovepress.com/oncotargets-and-therapy-journal agents and protocols on patient perspectives such as quality of life, adherence and satisfaction. The manuscript management system is completely online and includes a very quick and fair peer-review system, which is all easy to use. Visit http://www.dovepress.com/ testimonials.php to read real quotes from published authors. 